\title{
Deployment of Unmanned Systems after March 2011 Incident
}

Tim Trainer* *Robotic Products-International for iRobot's Defense \& Security business unit

\section{Background}

The Japanese earthquake and tsunami occurred in the middle of the night Boston-time on March 11, 2011, with large-scale damage from the event being broadcast the following morning. Japan is an important ally of the United States, and seeing the damage brought us great sorrow for the people of Japan.

\section{Prompt Decision to Donate Robotic Capa- bilities}

iRobot maintains a heritage of responding to largescale disaster and emergency response efforts. While conversations with Japanese authorities were not instantaneous, we communicated to our Japanese distributor that we were prepared to provide support as needed. We thought that our mid-sized multi-purpose robot 510 PackBot and large-size heavy duty robot 710 could help to investigate dangerous environments, including collapsed buildings and the Fukushima nuclear plant. Given the apparent need for support, iRobot decided to donate two of each robot for the response efforts.

\section{3. iRobot and Japan Relationship}

iRobot was founded by three roboticists from MIT, including our current CEO, Colin Angle, in 1990 to study, develop, produce and commercialize practical robots. The company is headquartered in Bedford, Massachusetts, a suburb of Boston, and maintains offices on the west coast of the United States, the United Kingdom, China and Hong Kong.

iRobot has a long history of providing robotic solutions to Japan. The autonomous vacuum cleaning

原稿受付 2014 年 1 月 4 日

キーワード : PackBot, 710, iRobot, Tim Trainer, Roomba

*8 Crosby Drive 12-2, Bedford, MA, USA robot Roomba is well known throughout Japan, and we have also provided underwater robots for oceanographic research. Roomba has undergone a series of improvements over the past several years to meet the demands of Japanese consumers for home appliances. iRobot has also exhibited its defense and security robots in the country and met with researchers to discuss robotic technologies.

\section{Overview of iRobot PackBot}

The iRobot 510 PackBot (Fig. 1) consists of a track drive chassis and flippers capable of maneuvering on various terrain and stairs. The chassis length is approximately $69[\mathrm{~cm}](89[\mathrm{~cm}]$ with the flippers extended) and its width is $53[\mathrm{~cm}]$ (with flippers). It is a modular design, allowing for the quick installation of various sensors depending on the mission need. PackBot has a three-link manipulator arm that can reach as high as $2[\mathrm{~m}]$. The configuration that was sent to Japan allows for remote surveillance, detection, identification, environmental monitoring, and lifting objects at the damaged facility. The arm is equipped with a gripper at the

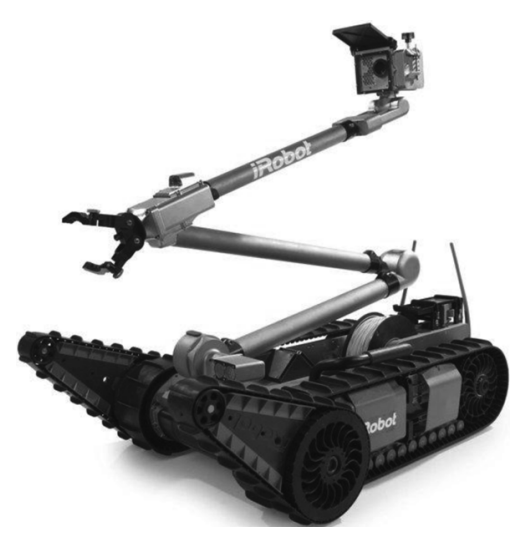

Fig. 1 The iRobot 510 PackBot provides first responders and other emergency personnel with situational awareness in dangerous scenarios 
joint between the 2 nd and 3 rd link. At the top of the 3rd link is pan/tilt mechanism for the main camera and lights. The gripper is used to handle explosives or dangerous substances. PackBot has both radio and wired communication modes and is operated using a ruggedized laptop PC and game-style hand controller. PackBot is powered by a lithium-ion rechargeable battery. One charge allows for 4-8 hours of operation.

The robot's interface is open to the US and international users, allowing for a variety of tools and accessories. Sensors are plug and play, and provide data to the laptop's display.

\section{Overview of iRobot $\mathbf{7 1 0}$}

The iRobot 710 system (Fig. 2) is a large and powerful robot with the ability to carry heavy loads, yet it is compact in size and highly mobile. In response to the 2011 earthquake and tsunami, iRobot provided two model 710 robots along with 2 of our smaller PackBot systems. As we were unsure what missions the 710 would be called upon to perform, one 710 was outfitted with a large manipulator arm and the other with a universal vice and hose clamp mount. The gripper for removal of debris and heavy lifting and the hose clamp mount to hold and maneuver a high capacity fire hose.

710 is a tracked vehicle like the 510 PackBot. It is relatively small and compact, yet very strong and highly maneuverable for a variety of tasks. The chassis consists of a track mechanism paired with flippers of the same size and a platform deck which provides for ex-

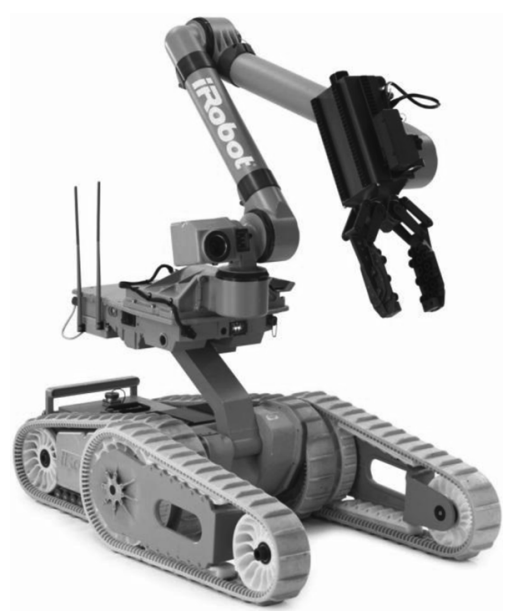

Fig. 2 The iRobot 710 is a powerful and rugged robot that carries heavy payloads, travels over rough terrain and climbs stairs while performing a variety of critical missions cellent mobility. The heavy manipulator arm, universal vice/hose clamp and other payloads were installed on the 710's large platform deck. The chassis length is approximately $89[\mathrm{~cm}]$ with the flippers folded and width is 54 [cm] (77 [cm] with flippers). 710 is a modular system like the 510 PackBot, providing the ability to install various payloads depending on the mission need. The robot's interface is open to US and international users allowing for easy integration of tools, end effectors and other payloads.

The heavy lift manipulator arm is a two-link design and is mounted to the 710's deck. The deck or platform lifts up and down, and tilts forward and aft, giving it a similar functionality as a three-link arm along with additional degrees of freedom. The arm is capable of lifting over $100[\mathrm{~kg}]$ and the gripper has a grip strength of 2,000 [N]. The deck also allows for the robot to shift its center of gravity, greatly improving maneuverability on stairs, over obstacles and other terrain. 710 weighs approximately $200[\mathrm{~kg}]$ and can travel up to $12[\mathrm{~km} / \mathrm{h}]$ with the arm installed.

Similar to 510 PackBot, 710 has radio and wired communication capabilities and is operated using a ruggedized laptop with game-style controller. The interface is very similar to 510 PackBot, allowing for shortened training time. However, the sheer weight and speed of 710 requires attention to detail and careful operation.

\section{Response Preparation for an Unknown De- ployment}

These robots have been used extensively to keep soldiers, law enforcement officers and first responders safe and to enhance operational success. Various tools, accessories, sensors and end effectors are provided for the platform, so the operator can be well equipped to perform a variety of tasks while maintaining a high degree of situational awareness. Because the deployment situation was unknown after the Great East Japan Earthquake, we decided to supply various accessories, tools and spares. As a severe accident at Fukushima was reported, we also decided to add sensors that can detect radiation levels, combustible gases and other environmental levels. A wealth of radio spares, batteries and various antenna and communication accessories were also provided.

The US Government approved the export of these robots in a rapid manner, and iRobot completed inven- 
tory, document verification, quality assurance and shipping preparation in a very short time. The 4 robotic systems were shipped out from Bedford on March 18, 5 days after the earthquake. Three iRobot employees departed Boston for Japan, along with three additional iRobot employees who were already in Asia, to support training.

\section{Delivery to TEPCO on March 24}

Upon arrival at Narita airport, iRobot employees departed for the cargo warehouse, staged the robots and confirmed functionality before delivering to TEPCO on March 24. Under a travel warning, we were unsure of transportation, so we met TEPCO for the first time at Tsuchiura. There, we conducted several hours of training. We have tremendous respect for TEPCO and its employees and their ability to receive the robots and complete training in English in such a short time and under such difficult circumstances.

Thousands of PackBots are used worldwide in difficult environments. Operating the robot with a game-style controller allows for quick training, and basic operation can be achieved within a short period of time. After opening the case, instructions for booting up the robot and operating modes are included in a brief manual. There is also a "help" display on the operator control unit to assist the user. A complete set of detailed instruction for operation, training and maintenance is also available. iRobot committed to fully supporting the operators that would be using these robots in Japan.

The 6 iRobot employees who made the trip to Japan stayed for two additional weeks to respond to TEPCO questions and to provide additional training and education about the various accessories that can be used with PackBot. Manuals for the Radiac(radiation sensor) and Combustible Gas sensors were also provided. As PackBot is often deployed in very dangerous environments, including the front lines of Iraq and Afghanistan, it is necessary for operators to completely understand how the robot functions, performance limits and how to utilize various sensors. We understand that TEPCO reviewed the operational scenario carefully and rehearsed extensively before entering Fukushima. This included communication verification testing as the reactor building is constructed of thick concrete with a large metallic object in the center.

iRobot conducted telephone conference calls with our
Japanese distributor every day beginning in March and every week thereafter until mid-August to resolve any issues. iRobot also continued to supply additional tools and accessories.

\section{Reactor Building Monitoring on April 17}

We received news that PackBot entered the reactor building and were grateful that they were helping in some way. It was broadcast that PackBot could not move into the reactor building \#2 due to high temperature and humidity levels, which caused condensation on the camera. Due to this, we received several offers for water repellent and hydrocolloid coating from all across Japan. With this fix implemented, the PackBot was able to re-enter building \#2 and verify that temperature, humidity and gas levels showed no immediate danger of an explosion and radiation levels showed as acceptable. This allowed for safe entry into reactor building \#2 by TEPCO workers.

While there were many communications challenges, working with TEPCO and other Japanese experts, we were able to establish reliable communications well into the facility. As TEPCO publicized, this allowed interior monitoring that revealed steam and high radiation concentrations of over $5[\mathrm{~Sv} / \mathrm{h}]$. Monitoring the situation via the robot to help reduce exposure to human operators.

\section{Maintenance and Robot Decontamination}

Robot decontamination is essential to reduce exposure to the operator and to sustain functionality. This can be achieved through low pressure water washdown of the systems. PackBot is waterproof, so this is ideal for such maintenance as decontamination of the system is critical for long term operations.

When customers order a PackBot, it comes with standard maintenance training and scheduled technical support visits. For the difficult circumstances surrounding the TEPCO, we reviewed and updated maintenance requirements through detailed manuals, illustrations and video to meet their requirements and ensure maximum system availability.

\section{Improvements to iRobot PackBot}

Stair climbing performance of the PackBot is qualified, however the severe slope angle of the stairs at Fukushima \#1 combined with oily water coating, scattered debris, and dust made stair climbing challenging. 
After carefully analyzing the metallic stair characteristics, iRobot tested and developed an improved track for better stair performance. The new tracks were provided to TEPCO, and improved performance and mobility were confirmed at the site. Ethernet cables and connectors were also replaced with environmentally hardened ones, improving reliability. However the handling of the cables did cause increased radiation exposure to the operator. In response, iRobot expedited the development of a mesh network and investigated connectivity with the TEPCO wireless network to reduce or eliminate the need for cabled operations.

\section{Improvements to iRobot $\mathbf{7 1 0}$}

It should be noted that the 710 was in the preproduction (production engineering) stage in March 2011. The systems we sent to Japan were literally taken from the engineers test benches. Since that time, improvements in hardware and software have been implemented. A bolt cutter, chain cutter and tool changers are also available for 710 . The interface has also been enhanced to support integration of Japanese systems including radios, sensors, end effectors and payloads.

\section{2. iRobot 710 with Vacuum Cleaner}

Use of the 710 for cleaning the reactor building entrance was publicized in June 2011. The robot was used to hold a dust collection nozzle and to pull the cleaner's hose. We saw it as the debut of the world's largest Roomba. The creation of this mission, along with its planning and application were highly admired. From this, we gained an understanding of potential additional applications, including the replacement of filters in nuclear facilities and removal of radioactive debris. 710 was also used at the Fukushima facility for interior debris removal.

\section{Japan Gamma Camera}

iRobot was asked to support the integration of a Japanese Gamma camera installation to allow for reactor building radiation mapping. While the majority of integration work was performed by Japanese companies, iRobot provided interface information under our standard developer kit and attended technical coordination meetings to verify functionality. It was noted that gamma camera system weight was approximately twice the qualified cargo load for PackBot. However by limiting speed of the system we were able to quickly certify the configuration for use in the facility, bringing another useful technology to bear on the problem.

\section{Collaboration}

Collaboration has been the key throughout TEPCO's operations in Fukushima. In addition to daily initial calls, an iRobot application engineer accompanied visits with the customer on numerous occasions and had technical discussions lasting well into the evening each time. We are honored to have played a small role in supporting the efforts at Fukushima. We acknowledge, respect and are grateful to TEPCO for achieving cold stabilization in a short period of time and for the robot operators for their commitment to the mission. Robots can save lives while performing dangerous tasks, and as we saw at Fukushima, can be used to reduce exposure and risk in a radioactive environment. I would like to acknowledge the US and Japanese governments for their support and give special thanks to iRobot colleagues and their families for their understanding and help.

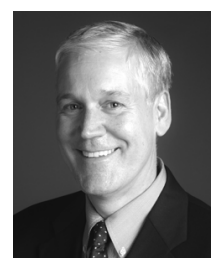

\section{Tim Trainer}

Tim Trainer is Vice President, Robotic Products-International for iRobot's Defense \& Security business unit. Prior to this appointment, he served as interim general manager and vice president of operations where he was responsible for all manufacturing, contracting and program management. Prior to that role, Trainer was vice president, programs and vice president, engineering. He joined iRobot after a 30 year career in the U.S. Navy, including positions as commanding officer of the Naval Aviation Depot (NADEP), North Island CA, head of Naval Air Systems Command 's Air Vehicle Engineering Department and various program positions. Trainer holds a bachelor's degree in mechanical engineering from Marquette University, a master's degree in aeronautical engineering from the Naval Postgraduate School in Monterey, California and he is a graduate of the U.S. Naval Test Pilot School in Patuxent River, Maryland. 